\title{
ADAPTIVE TECHNOLOGY FOR CONSTRUCTING THE KINETIC EQUATIONS OF REDUCTION REACTIONS UNDER CONDITIONS OF A PRIORI UNCERTAINTY
}

\author{
Dmitriy Demin $\bowtie$ \\ PC TECHNOLOGY CENTER \\ 4 Shatylova dacha str., Kharkiv, Ukraine, 61165 \\ dmitriydemin10@gmail.com \\ Oleh Domin \\ PE medical-diagnostic center «Arnica Plus», \\ 17-A Pushkina bulv., Kremenchuk, Ukraine, 39600
}

$\triangle$ Corresponding author

\begin{abstract}
The object of research is the process of oxide reduction in a reaction system of mass $m$ due to the reaction on a contact surface with an area of $S$.

An adaptive technology is proposed that allows one to construct the kinetic equation of the process in which the oxide is reduced from the initial product under conditions of a priori uncertainty.

A priori uncertainty regarding the behavior of a physicochemical system is understood as the fact that the following information is not available to the researcher:

- about the change in the mass of the reaction system and the area of the contact surface;

- about the rate of accumulation of the finished product;

- about the time of withdrawal of the finished product from the system.

The proposed adaptive technology includes five sequential stages to eliminate a priori uncertainty. This is ensured through the use of an adaptive algorithm, which allows obtaining the maximum accuracy in estimating the output variable by selecting the optimal parameter of the adaptive algorithm, and the subsequent canonical transformation. The introduced concept «canonical transformation of the kinetic equation» has the following meaning: having received some adequate description of the kinetic equation in a Cartesian coordinate system, a transformation is carried out that allow representing the equation in a new Cartesian coordinate system in such a way that its structure corresponds to the canonical form. The basic postulate of chemical kinetics can be such a canonical type.
\end{abstract}

Keywords: reaction system, system state, recovery process, adaptive algorithm, canonical transformation of the kinetic equation.

DOI: $10.21303 / 2461-4262.2021 .001959$

\section{Introduction}

Formal kinetics methods are still a reliable and relatively simple tool for studying physicochemical processes. The formalism of the approach to such a study of systems allows one to obtain adequate results due to the use of a rigorous mathematical apparatus for processing the observed experimental data. Obtaining mathematical models of the processes under study makes it possible to identify the mechanisms of these processes and, as a consequence, find ways to control them. This is evidenced by the publication of research results by methods of formal kinetics of processes that differ significantly in nature: combustion [1], flue gas cleaning [2], processes occurring during the processing of polymers (polyethylene processing) [3] and the production of explosive components [4], structural transformations in alloys [5], interactions between viral proteins and specific antibodies [6].

It should be noted that the methods of formal kinetics make it possible to obtain a mathematical description of processes not only in isolated or closed, but also in open systems, which, in fact, are the majority of industrial chemical technological systems. Therefore, it is natural to formalize such systems taking into account the internal chemical kinetics and mass transfer, which open up new possibilities in creating a wide range of chemical engineering products: catalysts [7], enriched 
minerals [8], coatings on materials [9], or obtaining new theoretical concepts on the processes for their subsequent implementation in the energy sector [10].

However, the peculiarities of some processes or conditions in which the physicochemical system functions require special approaches to the selection of data for the subsequent implementation of the procedure for estimating the rate constants and activation energies of the processes under study. Without this, the obtained results of calculating the kinetic parameters turn out to be inadequate. For example, in [11], devoted to the study of the kinetics of the formation of $\gamma$-lactone ( $\gamma$-lactone) during the processing of polyethylene in an open mixer, it was noted that the activation energy obtained by formal kinetics methods is more than 8 times lower than the experimental data demonstrate. As a solution to this problem, the authors of this work proposed a selection of the range of initial data in which the results obtained are adequate. Alternatives can be either the use of special experimental techniques [12], or the use of the theoretical principles of quantum chemistry [13]. In this regard, it is important to note that the use of such approaches is justified if there is a clear deviation of the processes under consideration from their linear description. The influence of the nonlinearity factor in the description of processes introduces significant difficulties into the process of building a model and requires special research methods, including mathematical ones. However, in the case of a successful construction of a model of the process under study, it becomes possible to search for its optimal control by initiating other physicochemical processes, the parameters of which serve as control actions. As an example, work [14] can be cited, in which, based on the construction of an adequate kinetic model, it is proposed to use the strategy of high-temperature deoxidation in place in the development of improved anodes for high-performance lithium-ion batteries. This has led to an acceleration of the electrochemical kinetics of $\mathrm{NiO}$, an increase in the efficiency of charge transfer, an increase in the speed of response, and provided a significant superiority in the characteristics of many NiO-based anodes.

Based on the above analysis, the following conclusion can be drawn: to obtain adequate kinetic models of physicochemical processes, taking into account its nature, one should take into account the potential effect of the range of experimental data on the result and the probable deviation from linearity in the description of the system. This requires the use of special research methods that make it possible to remove the uncertainty regarding the structure and methods of estimating the parameters of kinetic equations, which is one of the features of open systems in terms of formalizing their description.

In this paper, a technology is proposed that allows one to construct the kinetic equation of the process in which the oxide is reduced from the initial product under conditions of a priori uncertainty.

A priori uncertainty regarding the behavior of a physicochemical system is further understood as the fact that the following information is not available to the researcher:

- about the change in the mass of the reaction system in the physicochemical process occurring on the contact surface of the reductant and the reduced oxide, and the change in the area of the contact surface;

- about the rate of accumulation of the finished product;

- about the time of withdrawal of the finished product from the system.

In fact, a priori uncertainty leads to the fact that the researcher has access only to data on the concentrations of oxide in the initial product at control points in time.

\section{Materials and Methods}

\section{1. The object of research and its formal description}

The object of research is the oxide reduction process. The subject of research is a method of formal description of the process by the kinetic equation under conditions of a priori uncertainty.

Let there be a reaction system in which the initial product of mass $m$ contains $n \%$ of reducible oxide BO. For this, a reducing agent $\mathrm{A}$ is introduced into the system, as a result of which an interaction reaction of the form occurs on the contact surface with area $S$ : 


$$
(\mathrm{BO})+\mathrm{A}=[\mathrm{B}]+\mathrm{AO}
$$

where (BO) - concentration of oxide in the initial product, $\%$ by weight, $[\mathrm{B}]$ - the concentration of the finished product, $\%$ by weight.

If to take as the initial state of the system $s_{0}$ the state characterized by the initial mass of the initial product with the initial concentration of oxide, then as a result of this process, the mass of the initial product decreases due to the reduction of $\mathrm{BO}$ and the separation of the finished product B from the initial product. Thus, the system goes into state $s_{1}$.

In the future, four alternative options for the development of the process are possible, each of which can be characterized by a corresponding state:

$-s_{1}-$ a new portion of the original product is not introduced into the system and the finished product is not withdrawn from it;

$-s_{2}-$ the finished product is removed from the system, but a new portion of the original product is not introduced into the system;

$-s_{3}-$ the finished product is not removed from the system, but a new portion of the original product is introduced into the system;

$-s_{4}-$ the finished product is removed from the system and a new portion of the original product is introduced.

A schematic representation of the implementation of these processes in accordance with these four states is shown in Fig. 1.

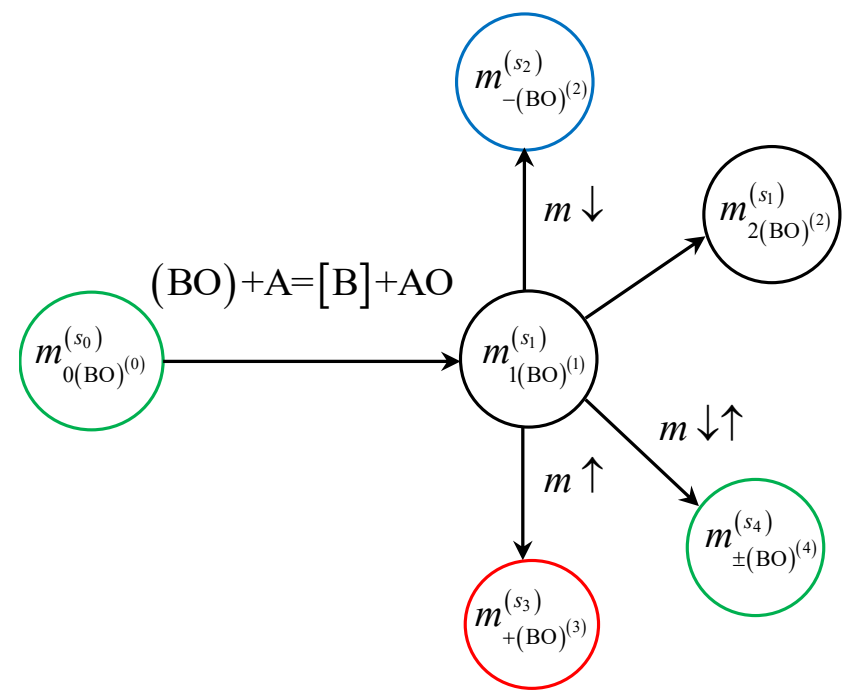

Fig. 1. Diagram of the system functioning process

In Fig. 1 the following designations are adopted:

$-m_{0(\mathrm{BO})^{(0)}}^{\left(s_{0}\right)}-$ mass of the initial product with the initial content of oxide $(\mathrm{BO})^{(0)}, \%$ by mass, corresponding to the initial state $s_{0}$;

$-m_{1(\mathrm{BO})^{(1)}}^{\left(s_{1}\right)}-$ mass of the starting product with the content of oxide $(\mathrm{BO})^{(1)}, \%$ by weight, as a result of the reaction (1), corresponding to the state $s_{1}, m_{1(\mathrm{BO})^{(1)}}^{\left(s_{1}\right)}=m_{0(\mathrm{BO})^{(0)}}^{\left(s_{0}\right)}-\Delta m^{-}$, where $\Delta m^{-}-$decrease in the mass of the starting product due to the course of reaction (1), leading to a change oxide concentration;

$-m_{2(\mathrm{BO})^{(2)}}^{\left(s_{1}\right)}$ - mass of the initial product with the oxide content $(\mathrm{BO})^{(2)}, \%$ by mass, as a result of the reaction (1), corresponding to the state $s_{1}, m_{2(\mathrm{BO})^{(2)}}^{\left(s_{1}\right)}=m_{1(\mathrm{BO})^{(1)}}^{\left(s_{1}\right)}-\Delta m^{-}$;

$-m_{-(\mathrm{BO})^{(2)}}^{\left(s_{2}\right)}$ - mass of the initial product with the oxide content $(\mathrm{BO})^{(2)}, \%$ by mass, as a result of the reaction (1) and the dispensing of a portion of product $\mathrm{B}$ from the system, corresponding to the state $s_{2}, m_{-(\mathrm{BO})^{(2)}}^{\left(s_{2}\right)}=m_{1(\mathrm{BO})^{(1)}}^{\left(s_{1}\right)}-m_{B}$, where $m_{B}$ - mass of the finished product B dispensed from the system; 
$-m_{+(\mathrm{BO})^{(3)}}^{\left(s_{3}\right)}-$ mass of the starting product with the content of oxide $(\mathrm{BO})^{(3)}, \%$ by weight, as a result of the reaction (1) and the introduction of a new portion of the starting product into the system, corresponding to the state $s_{3}, m_{+(\mathrm{BO})^{(3)}}^{\left(s_{3}\right)}=m_{1(\mathrm{BO})^{(1)}}^{\left(s_{1}\right)}+\Delta m^{+}$, where $\Delta m^{+}-$increase in the mass of the starting product introduced into the system ;

$-m_{ \pm(\mathrm{BO})^{(4)}}^{\left(s_{4}\right)}$ - mass of the initial product with the oxide content $(\mathrm{BO})^{(4)}, \%$ by mass, as a result of the reaction (1), the delivery of the finished product from the system and the compensation of the initial product by introducing a new portion of it into the system, corresponding to the state $s_{4}, m_{ \pm(\mathrm{BO})^{(4)}}^{\left(s_{4}\right)}=m_{1(\mathrm{BO})^{(1)}}^{\left(s_{1}\right)}-m_{B}+\Delta m^{+}$. If as a result compensation, the concentration of oxide in the original product returns to the original, then $m_{ \pm(\mathrm{BO})^{(4)}}^{\left(s_{4}\right)}=m_{ \pm(\mathrm{BO})^{(0)}}^{\left(s_{s}\right)}$;

$-m \downarrow-$ symbol of an event consisting in the issuance of a finished product with a mass $m_{B}$ from the system;

$-m \uparrow-$ symbol of an event consisting in the introduction into the system of a new portion of the initial product with a mass $\Delta m^{+}$;

$-m \downarrow \uparrow-$ symbol of an event consisting in issuing a finished product with a mass $m_{B}$ from the system, and compensating for the initial product by introducing a new portion of it with a mass of $\Delta m^{+}$into the system.

For ease of perception, the following color images have been introduced: green color - to describe the state $s_{0}$, blue color - to describe the state $s_{2}$, red color - to describe the state $s_{3}$.

The state $s_{4}$ actually reflects the fact that the process is regulated in some way, and there is an invariance with respect to the way of regulation.

In other words, the process can be controlled from the outside either by using technical devices (regulators), or by other physical and chemical processes that have a control effect on the system under consideration. This is the reason for the choice for the color display of the state $s_{4}$ in green, indicating that the process is being regulated in order to ensure the constancy of the initial state of the system.

However, it should be noted that regulation can be carried out not only with the aim of maintaining the constancy of the initial state, but also maintaining any other required state, depending on the chosen goal and the desired behavior of the system.

\section{2. Methods of research}

The kinetic equation was used to describe the process in general form:

$$
v=-\frac{d C}{d \tau}=\frac{k S}{m} C^{\eta}
$$

where $v$ - rate of the reduction process, \%/unit of time, $C=(\mathrm{BO}) / 100-$ concentration of $\mathrm{BO}$ in the initial product, $\tau$ - time, $\eta$ - order of the reaction with respect to oxide, $k$ is the rate constant of the process.

To determine the parameters of the kinetic equation (2), we used:

- an iterative procedure of adaptive mathematical modeling, which is based on the algorithm for calculating estimates of the coefficients of mathematical models in the conditions of non-additive drift [15], transformed into the form shown in Fig. 2, and hereinafter called the adaptive algorithm;

- transformation of Cartesian coordinates by parallel displacement of the axes in the coordinates $\tau-C$ and $C-v$;

- the method of least squares for constructing regression equations.

The following designations are adopted in Fig. 2:

$-\mathbf{F}_{N}$ - matrix of the experiment plan;

$-\mathbf{Y}$ - matrix of the output variable;

$-\delta-$ small positive value;

$-\alpha$ - time for which the forecast $y(\tau)$ is carried out;

$-h$ - given step;

$-r$ - value that takes values $0,1,2,3, \ldots$. 


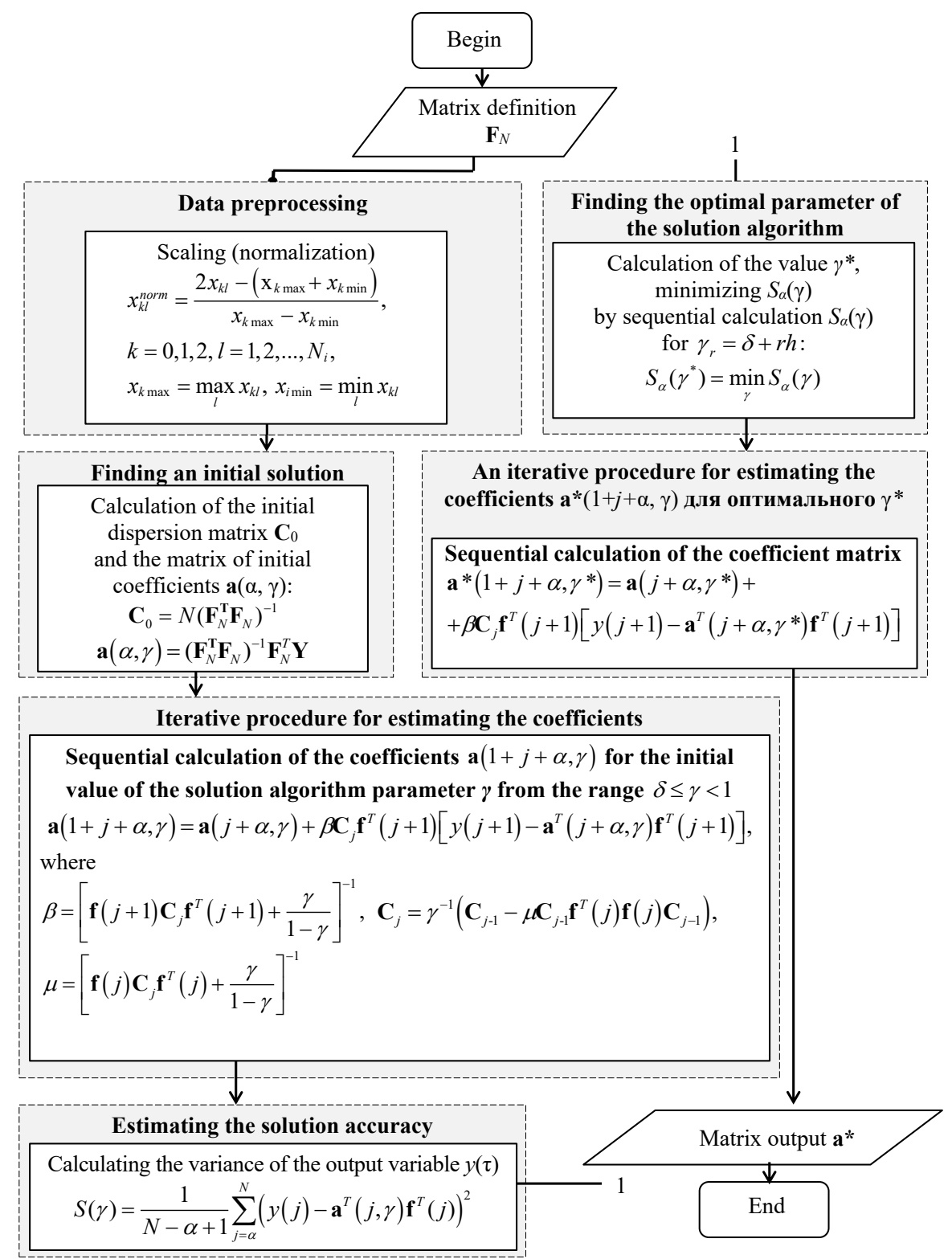

Fig. 2. Adaptive algorithm

\section{Results and discussion}

3. 1. The essence of the adaptive technology for constructing the kinetic equation: theoretical justification

The parameters $k$ and $\eta$ are unknown in equation (2), but under the conditions of a priori uncertainty, the parameters $S$, m are also unknown.

If over time there is a change in the mass of the initial product and the concentration of oxide in the product, not only during the course of reaction (1), but also due to the removal of the finished product from the system and the introduction of a new portion of the original product, then the rate of the reduction process will also change in time:

$$
v(\tau)=-\frac{d C(\tau)}{d \tau}
$$

This means that the result of determining the rate constant and the order of the reaction by linearizing equation (2) by the logarithm will be inadequate. The reason for this is the time-varying 
parameters of the equation, including abruptly at the moments of dispensing a portion of the reduction product and introducing a new portion of the original product. That is, the a priori uncertainty arising at the stage of data processing associated with the need to estimate the parameters of the kinetic equation only from the available data on the oxide concentrations does not make it possible to use the traditional approach to determine the parameters of the kinetic equation.

To remove this a priori uncertainty, an adaptive technology is proposed, which consists of five stages:

- stage 1: calculation and qualitative analysis of the instantaneous speeds of the process in order to identify the state $s_{i}$ and determine those experimental points that relate to this $i$-th state;

- stage 2: parallel shift of the axes in the Cartesian coordinates $\tau-C$ in order to bring the data to the state $s_{0}$;

- stage 3: implementation of stage 1 for the data reduced to the state $s_{0}$;

- stage 4: approximation of the experimental data reduced to the state $s_{0}$; in order to construct the kinetic equations $v=\varphi(C)$ for the states $s_{2}$ and $s_{3}$;

- stage 5: parallel shift of axes in Cartesian coordinates $C-v$ in order to bring the kinetic equations $v=\varphi(C)$ to a form similar in structure to equation (2), and approximation of the obtained data by the least squares method.

Since the operations of coordinate transformation are the basis for transforming the description of an object to some canonical form, it makes sense to introduce the concept of «canonical transformation of the kinetic equation». Such a definition has the following meaning: having some adequate description of the kinetic equation in the coordinate system in which such a description was obtained (the old coordinate system), a transformation is carried out that make it possible to represent the equation in the new coordinate system in such a way that its structure corresponds to some canonical mind. Such a canonical form can be, for example, form (2), which reflects the basic postulate of chemical kinetics.

Proceeding from this, stages 2, 4, 5 can actually be considered a canonical transformation of the kinetic equation, leading it to a canonical form in the sense of compliance with the basic postulate of chemical kinetics.

Stage 1. Having established at each time the rate of the oxide reduction process (hereinafter, for simplicity, referred to as the process rate), it is possible to identify the experimental points in the $\tau-C$ coordinates, which refer to different states $s_{i}(i=1,2,3)$.

The solution to equation (3) has the form:

$$
\int \mathrm{d} C(\tau)=-\int v(\tau) \mathrm{d} \tau
$$

or with the initial condition $\left.C\right|_{\tau=\tau_{0}=0}=C_{0}$ :

$$
C(\tau)=-v(\tau) \tau+C_{0}
$$

If to introduce into consideration the concept of the instantaneous rate of the process, understood as the rate of the process at the moment of time $\tau$, then equation (5) can be represented in the form:

$$
C_{j}(\tau)=-v_{j}(\tau) \tau
$$

which makes it possible to calculate the concentration of oxide $C_{j}$ at each moment of time $\tau$ using the obtained results of calculating the instantaneous velocities $v_{j}(\tau)$.

The graphic interpretation of the instantaneous velocities is as follows: the instantaneous velocity $\tau_{j}(\tau)$ is the tangent of the angle of inclination of the radius vector drawn from the origin to the point on the curve $C=f(\tau)$. Therefore, having the values of the instantaneous velocities, the general form of the dependence $C=f(\tau)$ can be recreated in the form (5), as a result of which the initial concentration $C_{0}$ can also be determined. 
The concentration values obtained by equation (6) can be called instantaneous concentrations. This interpretation of eq. (6) and the possibility of subsequent reconstruction of the general form of the dependence $C=f(\tau)$ make it possible to represent eq. (6) in the form:

$$
y_{j}(\tau)=a_{j}(\tau) \mathrm{d} \tau
$$

where $a_{j}(\tau)=v_{j}(\tau)$ - coefficient equal to the instantaneous rate of the process, $y_{j}(\tau)=C_{j}(\tau)$ - output variable equal to the instantaneous concentration.

The structure of equation (7) suggests that an adaptive algorithm can be used to calculate the coefficients $a_{j}(\tau)$ (Fig. 2). It should be noted that in this case the «-» sign indicating in equations (2)-(6) that the oxide is being consumed will be explicitly taken into account in solving equation (7).

The initial data for calculating instantaneous speeds by implementing an adaptive algorithm is given in the form of a Table $\mathbf{1}$.

It should be noted that in order to calculate the instantaneous velocities, the normalization operation may not be carried out, since only one independent variable is under consideration, and the further procedure requires knowledge of the instantaneous velocity in natural form. The results of the implementation of the adaptive algorithm are presented in the form of a Table 2.

Table 1

Initial data for calculating the instantaneous speeds of the process

\begin{tabular}{ccc}
\hline $\boldsymbol{\tau}$, time unit & $\boldsymbol{C}_{\boldsymbol{j}}(\boldsymbol{\tau}) \boldsymbol{\%}$ mass \\
\hline 0 & $C_{0}$ \\
$\tau_{1}$ & $C_{1}$ \\
$\tau_{2}$ & $C_{2}$ \\
$\tau_{3}$ & $C_{3}$ \\
$\cdot$ & $\cdot$ \\
$\tau_{N}$ & $C_{N}$
\end{tabular}

Table 2

Presentation of the results of the calculation of instantaneous speeds

\begin{tabular}{cc}
\hline $\boldsymbol{j}$ & $\boldsymbol{a}_{j}(\boldsymbol{\tau})=\boldsymbol{v}_{j}(\boldsymbol{\tau})=\boldsymbol{a}\left(\boldsymbol{\alpha}+\boldsymbol{j}, \boldsymbol{\gamma}^{*}\right)$ \\
\hline 0 & $a\left(\alpha, \gamma^{*}\right)$ \\
2 & $a\left(\alpha+1, \gamma^{*}\right)$ \\
3 & $a\left(\alpha+2, \gamma^{*}\right)$ \\
4 & $a\left(\alpha+3, \gamma^{*}\right)$ \\
$\mathbf{5}$ & $a\left(\alpha+4, \gamma^{*}\right)$ \\
6 & $\boldsymbol{a}\left(\boldsymbol{\alpha}+\mathbf{5}, \gamma^{*}\right)$ \\
7 & $a\left(\alpha+6, \gamma^{*}\right)$ \\
8 & $a\left(\alpha+7, \gamma^{*}\right)$ \\
$\mathbf{9}$ & $a\left(\alpha+8, \gamma^{*}\right)$ \\
. & $\boldsymbol{a}\left(\boldsymbol{\alpha}+\mathbf{9}, \gamma^{*}\right)$ \\
$N$ & $a\left(\alpha+N, \gamma^{*}\right)$ \\
$N+1$ & $a\left(\alpha+N+1, \gamma^{*}\right)$
\end{tabular}

The results are presented in the form of Table 2, refer to the optimal value of the parameter of the algorithm $\gamma^{*}$, which minimizes the estimate of the variance of the output variable $y(\tau)=C(\tau)$ according to the criterion $S_{\alpha}\left(\gamma^{*}\right)=\min _{\gamma} S_{\alpha}(\gamma)$.

The identifier of the event consisting in the transition from the state $s_{1}$ to the state $s_{2}$ or $s_{3}$ is an abrupt change in the instantaneous rate, which follows directly from the method for deter- 
mining the rate - a jump change in the oxide concentration due to the release of the finished product from the system or the introduction of a new portion of the initial product causes a significant change in the concentration after the same unit of time. Such values are conventionally highlighted in Table 2 in bold, and the lines corresponding to the transition between states are highlighted in different colors: blue - transition to state $s_{2}$, red - transition to state $s_{3}$. It should be noted that the transition to the state $s_{3}$ can also be carried out from the previous state $s_{2}$, and not only from the state $s_{1}$, as shown in Fig. 1, taking into account the continuity of the process.

The result of the calculations presented in Table 2 is the identification of system states.

Formally, other identifiers can be set by the implementation of the adaptive algorithm. For this, equation (2), taking into account (3), is transformed to the form:

$$
\ln v(\tau)=\ln \left(\frac{k(\tau) S(\tau)}{m(\tau)}\right)+\eta(\tau) \ln C(\tau)
$$

In this case, equation (8) can be represented as:

$$
y(\tau)=b_{0}(\tau) x_{0}+b_{1}(\tau) x_{1}(\tau)
$$

where $y(\tau)=\ln v(\tau), b_{0}(\tau)=\ln \left(\frac{k(\tau) S(\tau)}{m(\tau)}\right)$,

$$
x_{0}=1, b_{1}(\tau)=\eta(\tau), x_{1}(\tau)=\ln C(\tau),
$$

and the coefficients $b_{i}(\tau)$ for $i=0$ and $i=1$ can be determined using an adaptive algorithm. In this case, the experimental design matrix and the output variable matrix are specified as follows:

$$
\mathbf{F}_{N}=\left[\begin{array}{c}
1 f_{1}^{1}=x_{1}^{1} \\
1 f_{2}^{1}=x_{2}^{1} \\
1 f_{3}^{1}=x_{3}^{1} \\
\cdot \cdot \\
1 f_{N}^{1}=x_{N}^{1}
\end{array}\right], \mathbf{Y}=\left[\begin{array}{l}
y_{1} \\
y_{2} \\
y_{3} \\
\cdot \\
y_{N}
\end{array}\right],
$$

where the subscript corresponds to the experiment number and the superscript to the variable number.

If $x_{0}=1$ and $x_{1}$ are selected as input variables, respectively (9), then scaling is performed only in relation to $x_{1}$, which results in the translation of the value of $x_{1}$ into the dimensionless range $[-1 ; 1]$.

The results of the implementation of the adaptive algorithm with this choice of input variables and output variable are obtained in the coordinates $b_{0}(\tau)-b_{1}(\tau)$. The corresponding sets of values form points with coordinates $b_{0}(\tau), b_{1}(\tau)$ ), the location of which for different states forms clusters in the normalized space, which are separated depending on the state of the system $s_{i}$.

The transition from the normalized values $b_{0}(\tau)$ and $b_{1}(\tau)$ to natural values $a_{0}(\tau)$ and $a_{1}(\tau)$ is carried out by the formulas:

$$
\begin{gathered}
a_{0}=b_{0}-a_{1} \bar{x}_{1}, \\
a_{1}=\frac{b_{1}}{I},
\end{gathered}
$$

obtained by transforming the equation:

$$
y=a_{0} x_{0}+a_{1}\left(x_{1}^{\text {norm }} I+\bar{x}_{1}\right)
$$

where $\bar{x}_{1}$ - average value of the variable $x_{1}$ in the data sample, $\bar{x}_{1}=0.5\left(x_{1}^{\max }+x_{1}^{\min }\right), I=x_{1}^{\max }-\bar{x}_{1}=$ $=\bar{x}_{1}-x_{1}^{\mathrm{min}}$. 
It is important to note that the correct implementation of this procedure is possible only in the case of equality of time intervals, i. e., a constant value of the step of the argument $\tau$.

Separation of points $\left(b_{0}(\tau), b_{1}(\tau)\right)$ in the space of variables $b_{0}(\tau)-b_{1}(\tau)$ according to the obtained clusters is possible, for example, by parametric classification [16].

Stage 2. A parallel shift of the axes in the Cartesian coordinates $\tau-C$ in order to bring the data to the state $s_{0}$ is carried out according to the formulas:

$$
\begin{gathered}
\tau^{\prime}=\tau-\Delta \tau, \\
C^{\prime}=C-\Delta C,
\end{gathered}
$$

where $\Delta \tau$-value of the shift along the $\tau$ axis, chosen so that the oxide concentration after the transition to the $s_{2}$ state corresponds to the oxide concentration in the $s_{0}$ state.

Stage 3. For the data reduced to the state $s_{0}$, an adaptive algorithm is implemented similarly to stage 1 . The result is the calculation of the instantaneous velocities, but not for the general data sample, but for the data reduced to the state $s_{0}$, on the basis of which points with coordinates $\left(C^{\prime}, v^{\prime}\right)$.

Stage 4. The least squares method is used to calculate the estimates of the coefficients of the approximating equations from a set of points with coordinates $\left(C^{\prime}, v^{\prime}\right)$ in the space of variables $C^{\prime}-v^{\prime}$.

Stage 5. A parallel shift of the axes is carried out in Cartesian coordinates $C^{\prime}-v^{\prime}$ in order to bring the data to the state $s_{0}$ according to the formulas:

$$
\begin{gathered}
C^{\prime \prime}=C^{\prime}-\Delta C^{\prime}, \\
v^{\prime \prime}=v^{\prime}-\Delta v^{\prime},
\end{gathered}
$$

where $\Delta C^{\prime}, \Delta v^{\prime}$ - the amount of displacement along the axes $C^{\prime}$ and $v^{\prime}$, respectively. If the approximating equation has the form of a polynomial of the second degree (18), then the shifts are determined by calculating the extrema of the functions - approximating equations obtained by implementing stage 4 :

$$
\begin{gathered}
v^{\prime}=d_{0}+d_{1} C^{\prime}+d_{2} C^{2}, \\
\Delta C^{\prime}=-\frac{d_{1}}{2 d_{2}}, \\
\Delta v^{\prime}=d_{0}+d_{1} \Delta C^{\prime}+d_{2}\left(\Delta C^{\prime}\right)^{2} .
\end{gathered}
$$

The result of the implementation of the proposed adaptive technology is to obtain the kinetic equations in coordinates in the canonical form.

\section{2. Conditional practical example}

Let according to the results of observations of the system, the data given in Table 3. Between time points 4 and 5, the recovered product is dispensed from the system, and between time points 7 and 8 , a new portion of the original product is introduced into the system. This means that there is a transition between states $s_{1}, s_{2}, s_{3}$ (states $s_{2}$ and $s_{3}$ are marked in Table 3 in blue and red, respectively).

However, the researcher does not know about such processes in the system and to construct the kinetic equation is forced to operate only with the available data on the concentration of oxide in the initial product at control points in time.

In order for the obtained kinetic equation to adequately describe the process, it is necessary to implement five stages of adaptive technology. 
Table 3

Initial data

\begin{tabular}{ccc}
\hline Moment of time & $\boldsymbol{\tau}$, min & \multicolumn{1}{c}{$\boldsymbol{\%}$ mass } \\
\hline 1 & 0 & 0.38 \\
2 & 60 & 0.335 \\
3 & 120 & 0.3 \\
4 & 180 & 0.22 \\
5 & 240 & 0.145 \\
6 & 300 & 0.12 \\
7 & 360 & 0.14 \\
8 & 420 & 0.17 \\
9 & 480 & 0.15 \\
10 & 540 & 0.12
\end{tabular}

Stage 1 implementation. The results of calculating instantaneous velocities using the adaptive algorithm for the optimal value of the parameter $\gamma^{*}=10^{-5}\left(S_{\alpha}\left(\gamma^{*}\right)=4.49 \mathrm{E}-13\right)$ are given in Table 4.

Table 4

Results of calculating instantaneous speeds

\begin{tabular}{lc}
$\boldsymbol{j}$ & $\boldsymbol{v} \mathbf{. \% / \text { time unit }}$ \\
\hline 1 & 0.000667 \\
2 & 0.000833 \\
3 & 0.000333 \\
4 & $\mathbf{0 . 0 0 2 3 3 3}$ \\
5 & 0.000167 \\
6 & 0.000667 \\
7 & $\mathbf{0 . 0 0 1 3 3 3}$ \\
8 & 0.000333 \\
9 & 0.000333 \\
10 & 0.000667
\end{tabular}

From Table 4, it can be seen that at times 4 and 7 the rate of the process changed abruptly, which indicates that in the interval between the previous and the next with respect to times 4 and 7, there was an abrupt change in the mass of the system. If this is not taken into account, then the use of the traditional approach to the construction of the kinetic equation will give an inadequate result.

Based on the established fact of change in the mass of the system, the entire data sample can be divided into three classes, corresponding to the states $s_{0}, s_{2}, s_{3}$.

When using equation (9), the initial data are reduced to the form shown in Table 5.

The results of calculating the parameters $b_{0}(\alpha+j, \gamma)$ and $b_{1}(\alpha+j, \gamma)$ using the adaptive algorithm for the optimal value of the parameter $\gamma^{*}=10^{-3}\left(S_{\alpha}\left(\gamma^{*}\right)=3.55845 \mathrm{E}-11\right)$ are given in Tables 6-8.

in Table 7.

Recalculation of parameters from normalized to natural form leads to the results shown 
Table 5

Initial data for the implementation of the adaptive algorithm in the case of using equation (9)

\begin{tabular}{cccc}
\hline $\boldsymbol{\tau}$ & \multicolumn{2}{c}{$\boldsymbol{F}$} & $\boldsymbol{y}(\boldsymbol{\tau})$ \\
\cline { 2 - 4 } & $\boldsymbol{x}_{\mathbf{1}}(\boldsymbol{\tau})$ & $\boldsymbol{x}_{\mathbf{2}}(\boldsymbol{\tau})$ & -7.31322 \\
2 & 1 & 1 & -7.09008 \\
3 & 1 & 0.824987 & -8.00637 \\
4 & 1 & 0.671765 & -6.06046 \\
5 & 1 & 0.241102 & -8.69951 \\
6 & 1 & -0.33777 & -7.31322 \\
7 & 1 & -0.60054 & -6.62007 \\
8 & 1 & -0.3865 & -8.00637 \\
9 & 1 & -0.1169 & -8.00637 \\
10 & 1 & -0.2907 & -7.31322
\end{tabular}

Table 6

The results of calculating the parameters $b_{0}(\alpha+j, \gamma)$ and $b_{1}(\alpha+j, \gamma)$ in the case of using equation (9)

\begin{tabular}{|c|c|c|c|c|c|c|}
\hline$j$ & $b_{0}\left(\alpha+j, \gamma^{*}\right)$ & $b_{1}\left(\alpha+j, \gamma^{*}\right)$ & $y_{\text {calc }}$ & $y_{\text {exp }}$ & $\Delta y$ & $(\Delta y)^{2}$ \\
\hline 0 & -7.4498 & 0.170694 & & & & \\
\hline 1 & -7.45765 & 0.144438 & -7.31321 & -7.31322 & $-8.92 \mathrm{E}-06$ & $7.96 \mathrm{E}-11$ \\
\hline 2 & -6.07836 & -1.22636 & -7.09009 & -7.09008 & $9.82 \mathrm{E}-06$ & $9.64 \mathrm{E}-11$ \\
\hline 3 & -12.0116 & 5.962269 & -8.00637 & -8.00637 & $-1.48 \mathrm{E}-06$ & $2.18 \mathrm{E}-12$ \\
\hline 4 & -4.97229 & -4.51334 & -6.06046 & -6.06046 & $5.73 E-07$ & $3.28 E-13$ \\
\hline 5 & -7.1636 & 4.547193 & -8.69951 & -8.69951 & $-2.90 E-06$ & $8.43 E-12$ \\
\hline 6 & -10.4401 & -5.20683 & -7.31323 & -7.31322 & $1.24 \mathrm{E}-05$ & $1.55 \mathrm{E}-10$ \\
\hline 7 & -5.36936 & 3.236001 & -6.62007 & -6.62007 & $2.75 E-06$ & $7.57 E-12$ \\
\hline 8 & -8.60608 & -5.13015 & -8.00637 & -8.00637 & $-1.42 E-06$ & $2.01 E-12$ \\
\hline 9 & -8.00765 & -0.00441 & -8.00637 & -8.00637 & $-2.15 \mathrm{E}-06$ & 4.63E-12 \\
\hline 10 & -8.65553 & -2.23517 & -7.31322 & -7.31322 & $2.17 \mathrm{E}-07$ & $4.72 \mathrm{E}-14$ \\
\hline
\end{tabular}

Note: the lower and upper boundaries of the intervals $j$, which form the time intervals at which the transition between the states of the system, are marked with oblique font.

Table 7

The results of calculating the parameters $a_{0}\left(\alpha+j, \gamma^{*}\right)$ and $a_{1}\left(\alpha+j, \gamma^{*}\right)$ in the case of using equation (8) in natural form

\begin{tabular}{ccc}
\hline $\boldsymbol{j}$ & $\boldsymbol{a}_{\mathbf{0}}\left(\boldsymbol{\alpha}+\boldsymbol{j}, \boldsymbol{\gamma}^{*}\right)$ & $\boldsymbol{a}_{\mathbf{1}}\left(\boldsymbol{\alpha}+\boldsymbol{j}, \boldsymbol{\gamma}^{*}\right)$ \\
\hline 0 & -7.04978 & 0.237015 \\
1 & -7.11916 & 0.200557 \\
2 & -8.95235 & -1.70284 \\
3 & 1.961097 & 8.278849 \\
4 & -15.5494 & -6.26695 \\
5 & 3.492845 & 6.313959 \\
6 & -22.6425 & -7.2299 \\
7 & 2.21428 & 4.493316 \\
8 & -20.6287 & -7.12342 \\
9 & -8.018 & -0.00613 \\
10 & -13.8937 & -3.10362
\end{tabular}

Note: the lower and upper boundaries of the intervals $j$, which form the time intervals at which the transition between the states of the system, are marked with oblique font. 
The results of data separation by states in coordinates $b_{0}\left(\alpha+j, \gamma^{*}\right)-b_{1}\left(\alpha+j, \gamma^{*}\right)$ are shown in Fig. 3.

Fig. 3 that the presence of discontinuities in the function $C=f(\tau)$ leads to poor separation of classes by states. To improve the accuracy, it is necessary to exclude from consideration the extreme points for the states $s_{1}-S_{2}$ and $s_{2}-S_{3}$.

In this example, the worst case is taken - for a small sample of data, which does not allow excluding the extreme points. In the case of a larger sample of data, a higher classification accuracy should be expected.

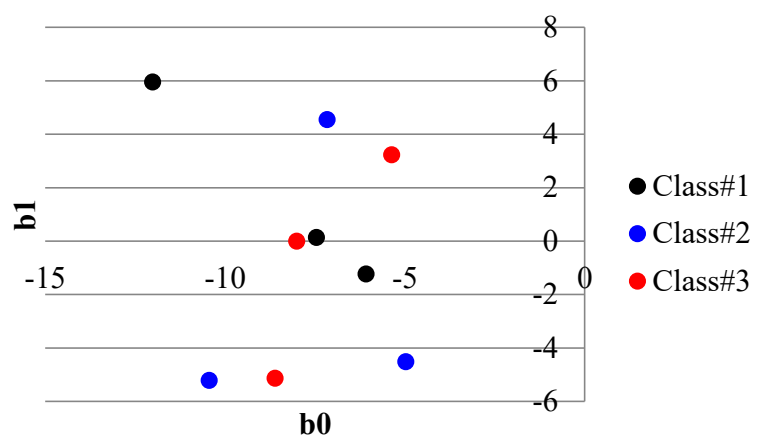

Fig. 3. Distribution of points $\left(b_{0}, b_{1}\right)$ by states

Stage 2 implementation. The results of calculating the displacement along the axes in the coordinates $\tau-C$ are presented in Table 8 .

Table 8

Results of calculating the displacement along the axes in the coordinates $\tau-C$

\begin{tabular}{|c|c|c|c|c|c|}
\hline \multicolumn{2}{|c|}{$s_{0}$} & \multicolumn{2}{|c|}{$s_{2}$} & \multicolumn{2}{|c|}{$s_{3}$} \\
\hline$\Delta \tau$ & $\Delta C$ & $\Delta \tau$ & $\Delta C$ & $\Delta \tau$ & $\Delta C$ \\
\hline 0 & 0.38 & 240 & 0.235 & 420 & 0.21 \\
\hline
\end{tabular}

The results of the data reduction to the state $s_{0}$ are presented in Table 9.

Table 9

Results of data reduction to the state $s_{0}$

\begin{tabular}{cc}
\hline $\boldsymbol{\tau}^{\prime}, \boldsymbol{m i n}$ & $\boldsymbol{C}^{\prime} \boldsymbol{\prime}$ \% mass \\
\hline 0 & 0.38 \\
60 & 0.335 \\
120 & 0.3 \\
0 & 0.38 \\
60 & 0.355 \\
60 & 0.38 \\
120 & 0.36
\end{tabular}

Stage 3 implementation. The results of calculating the instantaneous velocities for the data reduced to the state $s_{0}$ are presented in Table $\mathbf{1 0 .}$ 
Table 10

The results of calculating the instantaneous velocities for the data reduced to the state $s_{0}$

\begin{tabular}{ccc}
\hline $\boldsymbol{\tau}^{\prime}, \boldsymbol{m i n}$ & $\boldsymbol{C}^{\prime}, \boldsymbol{\%}$ mass & $\boldsymbol{v}^{\prime}, \boldsymbol{\%} / \mathbf{m i n}$ \\
\hline 0 & 0.38 & -0.00075 \\
60 & 0.335 & -0.00058 \\
120 & 0.3 & 0.0025 \\
0 & 0.38 & -0.00042 \\
60 & 0.355 & 0.005917 \\
0 & 0.38 & -0.00033 \\
60 & 0.36 & -0.0005 \\
120 & 0.33 & 0.00275
\end{tabular}

Stage 4 implementation. Fig. 4 shows the results of the approximation of the data obtained on the basis of Table 10, in the space of variables $C^{\prime}-v^{\prime}$.

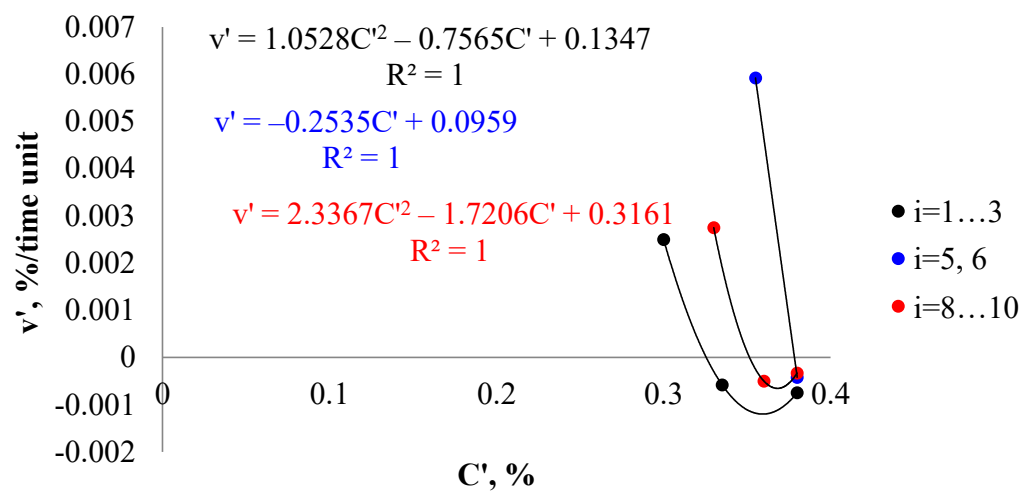

Fig. 4. Kinetic equations in the form of a polynomial of the second degree

Stage 5 implementation. The displacement values along the coordinate axes are determined by formulas (18), (19) and are given in Table 11.

The data are presented only for the states $s_{0}$ and $s_{3}$ due to the lack of a sufficient number of points for the state $s_{2}$

Table 11

The results of calculating the shift along the axes in coordinates $C^{\prime}-v^{\prime}$

$s_{0}$

$s_{3}$

\begin{tabular}{cccc}
\hline$\Delta \boldsymbol{C}^{\prime}$ & $\Delta \boldsymbol{v}^{\prime}$ & $\Delta \boldsymbol{C}^{\prime}$ & $\Delta \boldsymbol{v}^{\prime}$ \\
\hline 0.35928 & -0.0012 & 0.368169 & -0.00064
\end{tabular}

The results of constructing kinetic equations in canonical form are presented in Table 12 and Fig. 5, 6.

As a result, the kinetic equations were obtained in canonical form in the coordinates $C^{\prime \prime}-v^{\prime \prime}$ :

$$
\begin{aligned}
& v^{\prime \prime}=1.0528 C^{\prime \prime 2}, \\
& v^{\prime \prime}=2.3367 C^{\prime \prime 2} .
\end{aligned}
$$


Table 12

Results of the canonical transformation for constructing the canonical equation in the coordinates $C^{\prime \prime}-v^{\prime \prime}$

\begin{tabular}{cccc}
\hline & \multicolumn{1}{c}{$\boldsymbol{s}_{\mathbf{0}}$} & $\boldsymbol{s}_{\mathbf{3}}$ & $\boldsymbol{v}^{\prime \prime}$ \\
\hline $\boldsymbol{C}^{\prime \prime}$ & $\boldsymbol{v}^{\prime \prime}$ & $\boldsymbol{C}^{\prime \prime}$ & 0.000306 \\
\hline 0.02072 & 0.000448 & 0.011831 & 0.000136 \\
-0.02428 & 0.000618 & -0.00817 & 0.003386
\end{tabular}

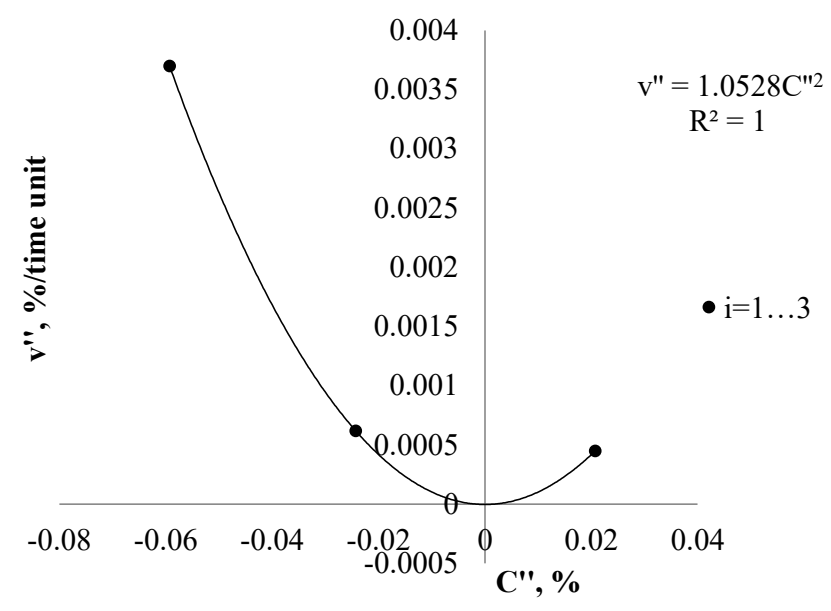

Fig. 5. The results of constructing the kinetic equation in the canonical form for the state $s_{0}$

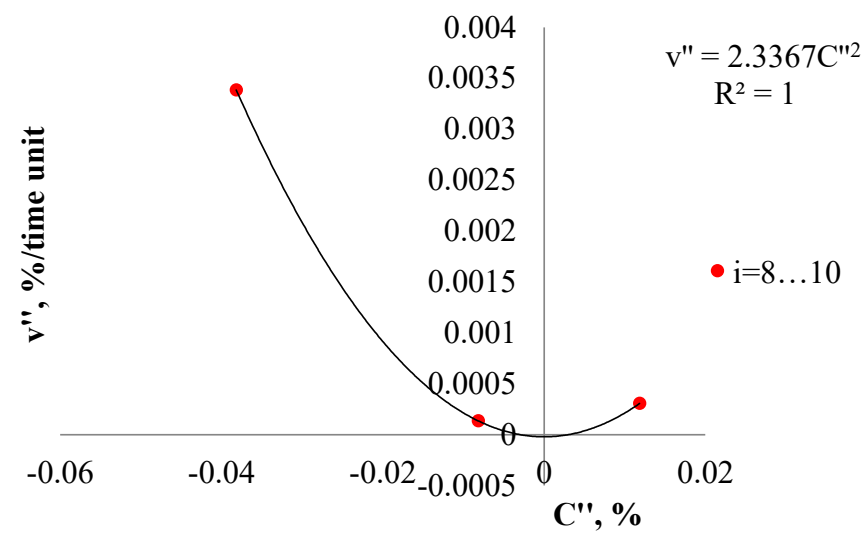

Fig. 6. Results of constructing the kinetic equation in the canonical form for the state $s_{3}$

To achieve the state $s_{4}$, it is necessary to additionally obtain a description of the behavior of the system in the classical representation of control theory, since in fact we are talking about the synthesis of a controller. The difficulties arising in this case, associated, for example, with a nonlinear description of the system, can be overcome artificially if there is a fundamental possibility of reducing the descriptions of the system to the form for which there are well-developed solutions for the synthesis of the controller [17, 18].

It is important to note that the proposed technology requires the use of the same time step, and the results obtained depend on the correctly chosen structure of the kinetic model. This is a limitation on the use of technology in general. If the structure of the kinetic equation really corresponds to the form (2), then the coefficient in the equation of the canonical form in the coordinates $C^{\prime \prime}-v^{\prime \prime}$ corresponds to the value $k S / m$. Therefore, to determine the three unknown 
parameters of the equation $k, S, m$ it is sufficient to have two additional equations. A variant of reducing the dimension of the problem arising in this case can be the calculation of the value of the decrease in the amount of the initial product for each of the states reduced to the initial state $s_{0}$, and the rate according to the known formulas:

$$
\begin{gathered}
\Delta m(\tau)=m_{0} \frac{C_{0}-C(\tau)}{1-C(\tau)}, \\
v=-\frac{d C(\tau)}{d \tau}=k(\tau) S(\tau) \frac{(1-C(\tau))^{2} C^{\eta}}{m_{0}\left(1-C_{o}\right)},
\end{gathered}
$$

where $\Delta m(\tau)$ - decrease in the mass of the initial product at the moment of time $\tau$ due to the occurrence of reaction (1), corresponds to the value of $\Delta m^{-}$in Fig. 1, but as a function of time.

The development of a procedure for estimating unknown parameters in general terms can be a direction for further research.

\section{Conclusions}

The implementation of the proposed adaptive technology, which includes five sequential stages, makes it possible to remove the a priori uncertainty in the estimation of the parameters of the kinetic equations describing the reduction reactions in a physicochemical system proceeding on a certain contact surface. This is ensured through the use of an adaptive algorithm, which allows obtaining the maximum accuracy in estimating the output variable by selecting the optimal parameter $\gamma^{*}$ of the adaptive algorithm, and the subsequent canonical transformation. This transformation brings the kinetic equation to a canonical form in the sense of conformity to the basic postulate of chemical kinetics.

\section{References}

[1] Khichar M., Thynell, S. T. (2021). A reduced mechanism with optimal rate-kinetics parameters for liquid-phase decomposition of bis(triaminoguanidinium) 5,5'-azotetrazolate (TAGzT): Quantum chemical calculations, thermolysis experiments and kinetic modeling. Thermochimica Acta, 699, 178895. doi: http://doi.org/10.1016/j.tca.2021.178895

[2] Chen, S., Qin, C., Yin, J., Zhou, X., Chen, S., Ran, J. (2021). Understanding sulfation effect on the kinetics of carbonation reaction in calcium looping for CO2 capture. Fuel Processing Technology, 221, 106913. doi: http://doi.org/10.1016/j.fuproc.2021.106913

[3] Gugumus, F. (2006). Physico-chemical aspects of polyethylene processing in an open mixer. Part 26: Formal kinetics of aldehyde and carboxylic acid formation at a constant rate. Polymer Degradation and Stability, 91 (11), $2698-2714$. doi: http://doi.org/10.1016/j.polymdegradstab.2006.04.021

[4] Gao, X., Jiang, L., Xu, Q., Wu, W.-Q., Mensah, R. A. (2020). Thermal kinetics and reactive mechanism of cellulose nitrate decomposition by traditional multi kinetics and modeling calculation under isothermal and non-isothermal conditions. Industrial Crops and Products, 145, 112085. doi: http://doi.org/10.1016/j.indcrop.2020.112085

[5] Marceaux dit Clément, A., Hoummada, K., Drillet, J., Hébert, V., Maugis, P. (2020). Effects of cementite size and chemistry on the kinetics of austenite formation during heating of a high-formability steel. Computational Materials Science, $182,109786$. doi: http://doi.org/10.1016/j.commatsci.2020.109786

[6] Plikusiene, I., Maciulis, V., Ramanaviciene, A., Balevicius, Z., Buzavaite-Verteliene, E., Ciplys, E. et. al. (2021). Evaluation of kinetics and thermodynamics of interaction between immobilized SARS-CoV-2 nucleoprotein and specific antibodies by total internal reflection ellipsometry. Journal of Colloid and Interface Science, 594, 195-203. doi: http://doi.org/10.1016/ j.jcis. 2021.02 .100

[7] Salmi, T., Russo, V., Freites Aguilera, A. (2021). Modelling of the interaction of kinetics and external transport phenomena in structured catalysts: The effect of reaction kinetics, mass transfer and channel size distribution in solid foams. Chemical Engineering Science, 244, 116815. doi: http://doi.org/10.1016/j.ces.2021.116815

[8] Vaziri Hassas, B., Kouachi, S., Eskanlou, A., Bouhenguel, M., Çelik, M. S., Miller, J. D. (2021). The significance of positive and negative inertial forces in Particle-Bubble interaction and their role in the general flotation kinetics model. Minerals Engineering, 170, 107006. doi: http://doi.org/10.1016/j.mineng.2021.107006

[9] Rozanov, L. N. (2021). Kinetic equations of non-localized physical adsorption in vacuum for Freundlich adsorption isotherm. Vacuum, 189, 110267. doi: http://doi.org/10.1016/j.vacuum.2021.110267 
[10] Leite, V. B., Kalempa, D., Graur, I. (2021). Kinetic modelling of evaporation and condensation phenomena around a spherical droplet. International Journal of Heat and Mass Transfer, 166, 120719. doi: http://doi.org/10.1016/j.ijheatmasstransfer. 2020.120719

[11] Gugumus, F. (2007). Physico-chemical aspects of polyethylene processing in an open mixer. Part 30: Formal kinetics of $\gamma$-lactone formation at a constant rate. Polymer Degradation and Stability, 92 (1), 158-175. doi: http://doi.org/10.1016/ j.polymdegradstab.2006.09.010

[12] Ma, S., Yang, M., Pang, S., Zhang, Y. (2021). Subsecond measurement on deliquescence kinetics of aerosol particles: Observation of partial dissolution and calculation of dissolution rates. Chemosphere, 264, 128507. doi: http://doi.org/ 10.1016/j.chemosphere.2020.128507

[13] Sanches-Neto, F. O., Ramos, B., Lastre-Acosta, A. M., Teixeira, A. C. S. C., Carvalho-Silva, V. H. (2021). Aqueous picloram degradation by hydroxyl radicals: Unveiling mechanism, kinetics, and ecotoxicity through experimental and theoretical approaches. Chemosphere, 278, 130401. doi: http://doi.org/10.1016/j.chemosphere.2021.130401

[14] Peng, J., Zhang, W., Zheng, M., Hu, H., Xiao, Y., Liu, Y., Liang, Y. (2021). Propelling electrochemical kinetics of transition metal oxide for high-rate lithium-ion battery through in situ deoxidation. Journal of Colloid and Interface Science, 587, 590-596. doi: http://doi.org/10.1016/j.jcis.2020.11.016

[15] Hartman, K., Leckiy, E., Shefer, V. et. al. (1997). Planirovanie ehksperimenta v issledovanii tekhnologicheskih processov. Moscow: Mir, 552.

[16] Demin, D. (2020). Constructing the parametric failure function of the temperature control system of induction crucible furnaces. EUREKA: Physics and Engineering, 6, 19-32. doi: http://doi.org/10.21303/2461-4262.2020.001489

[17] Demin D. A. (2014). Mathematical description typification in the problems of synthesis of optimal controller of foundry technological parameters. Eastern-European Journal of Enterprise Technologies, 1 (4 (67)), 43-56. doi: http://doi.org/10.15587/ 1729-4061.2014.21203

[18] Demin, D. A. (2012). Synthesis of optimal temperature regulator of electroarc holding furnace bath. Naukovyi Visnyk Natsion-alnoho Hirnychoho Universytetu, 6, 52-58.

How to cite: Demin, D., Domin, O. (2021). Adaptive technology for constructing the kinetic equations of reduction reactions under conditions of a priori uncertainty. EUREKA: Physics and Engineering, 4, 14-29. doi: http://doi.org/10.21303/ 2461-4262.2021.001959 